\title{
Numerical Simulation of Interaction of Sonic Jet with High Speed Flow over a Blunt Body using Solution Mapped Higher Order Accurate AUSM+-UP Based Flow Solver
}

\author{
P. Nair ${ }^{1}$, T. Jayachandran ${ }^{1}$, M. Deepu ${ }^{2}$, B.P. Puranik ${ }^{3}$ and U.V. Bhandarkar ${ }^{3}$ \\ ${ }^{I}$ FMTD/PRG/VSSC, ISRO, Thiruvananthapuram-695 022, India. \\ ${ }^{2}$ Dept. of Aerospace Engg; Indian Institute of Space Science and Technology, Thiruvananthapuram-695 022, India. \\ ${ }^{3}$ Department of Mechanical Engineering, Indian Institute of Technology Bombay, India 440076
}

Email:scientist2k@yahoo.com

(Received October 3, 2008; accepted March 28, 2009)

\begin{abstract}
The development of a numerical procedure based on AUSM+-UP scheme using higher order accurate reconstruction method is presented. A code based on this is used for the simulation of film cooling for reentry module. Here the convective fluxes are evaluated using AUSM+-UP scheme. Least square based derivative evaluation is used to compute diffusive fluxes. The numerical code has been successfully validated using standard experimental data for counter flow injection. Analysis has been carried out for a simple axisymmetric reentry module with and without film cooling, for a free stream Mach number of 8.0. The predicted adiabatic wall temperatures were compared for both the cases. Film cooling is found to be effective for this configuration and injected coolant remains confined to the boundary layer formed by the free stream from nose tip to the aft end of the module. Numerical simulation of film cooling provides vital information required for design of effective cooling system such as number of counter flow injectors, their dimensions and locations, injection pressure and temperature, mass flow rate required etc.
\end{abstract}

Keywords: Higher order reconstruction, hypersonic flow, sonic jet, film cooling, Finite Volume Method, AUSM+UP schemes.

\section{NOMENCLATURE}

$\begin{array}{ll}C_{p} & \text { Specific heat at constant pressure }(\mathrm{kJ} / \mathrm{kgK}) \\ C_{\mu} & \text { Closure coefficient } \\ E & \text { Total energy }(\mathrm{kJ}) \\ E_{i j} & \text { Deformation tensor } \\ F \& G & \text { Flux vectors } \\ K & \text { Thermal conductivity }\left(\mathrm{W} / \mathrm{m}^{2} \mathrm{~K}\right) \\ N_{n} & \text { Number of neighbours in the stencil } \\ \mathrm{n} & \text { Order of accuracy } \\ P r & \text { Prandtl Number } \\ Q & \text { Heat flux }\left(\mathrm{W} / \mathrm{m}^{2}\right) \\ R & \text { Residue/ universal gas constant } \\ S & \text { Source term } \\ U & \text { Vector of conservative variables }\end{array}$

\section{INTRODUCTION}

The current trend in the development of upwind schemes is based on the hybrid flux splitting schemes. This combines the accuracy of finite difference schemes in the resolution of the boundary layer and the robustness of finite volume schemes in the capturing of

$\begin{array}{ll}u & \text { Velocity in x direction }(\mathrm{m} / \mathrm{s}) \\ V & \text { Volume of the control volume }\left(\mathrm{m}^{3}\right) \\ v & \text { Velocity in y direction }(\mathrm{m} / \mathrm{s}) \\ x \& y & \text { Cartesian coordinates } \\ Y & \text { Mass fraction of a chemical species } \\ \varepsilon & \text { Kinetic energy dissipation rate } \\ \varepsilon_{j} & \text { Error in reconstruction } \\ \Phi & \text { Primitive variable } \\ \kappa & \text { Kinetic energy of turbulence }(\mathrm{kJ}) \\ \mu & \text { Dynamic viscosity }(\mathrm{kg} / \mathrm{m} . \mathrm{s}) \\ \rho & \text { Density }\left(\mathrm{kg} / \mathrm{m}^{3}\right) \\ \sigma & \text { Shear stress }\left(\mathrm{N} / \mathrm{m}^{2}\right) \\ \omega & \text { Geometric weight }\end{array}$

strong discontinuities. One of the most remarkable hybrid flux splitting schemes is Liou and Steffen's Advection Upstream Splitting Method (AUSM) as reported by Liou (1996). In this scheme, the inviscid flux at a cell interface is split into convective flux, upwinded in the direction of the flow, and pressure flux, upwinded on acoustic considerations. As a result, AUSM can capture the boundary layer quite accurately, 
even with $1^{\text {st }}$ order spatial accuracy. The present AUSM+-UP scheme have additional features such as exact resolution of contact and shock discontinuities, positivity preserving of scalar quantity such as the density and is free of "carbuncle" phenomena. This computationally effective scheme can be easily extended for other hyperbolic systems and is free of oscillations at the slowly moving shocks.

Atmospheric vehicle traveling at very high speeds experiences aerodynamic heating. This can be critical to the missions as in the case of re-entry vehicles. Strong shocks around the body increases the temperature to such a high value that chemical reactions like ionization, dissociation etc occur and results in communication blackout. Use of aerodynamically blunt body for reentry and hypersonic applications reduces the thermal loads on the module. Various ways of alleviating the problems due to this are accordingly of great interest. The methods usually adopted for thermal protection can be broadly classified into active and passive techniques (David and James, 1963). In passive systems, protective surfaces like silica or carbon tiles, capable of withstanding high temperatures, are introduced. Sometimes ablatives are used in regions where high temperature gradients are expected. On the other hand, in active systems the flow field around the re-entry body is altered thereby reducing the pressure drag and heat flux. Important techniques which come under this category are film cooling/counter flow jet, aerospike/aerodisc, aerospike with opposing jet and cavity technique. All these methods are illustrated by Sakagoshi and Ken (2000).The opposing jet method consists of an upstream-directed jet (cold gas) emanating from the stagnation region of the body. The coolant flow is then diverted rearward by the action of the incoming stream and the gas by flowing back over the body forms a heat buffer between the hot stream and the body. This can alter the physical properties in a buffer layer immediately adjacent to the body. In the case of aerospike concept, the spike forms an integral part at the fore end of the body and is effective in reducing the aerodynamic drag and heat transfer rates on axisymmetric blunt bodies in supersonic/hypersonic flow. In cavity technique minute cavities are generated on nose cone tip to ensure proper entrapment of cold fluid at the nose tip.

In the present work, flow over a typical reentry module has been numerically simulated using finite volume based flow solver. Convective fluxes are evaluated using AUSM+-UP scheme and diffusive fluxes using least square based derivative evaluation. Turbulence was modeled using RNG k- $\varepsilon$ model. Time integration was performed explicitly using 3-stage Runga-Kutta scheme.

\section{Physics OF The Problem}

Many experimental tests are reported earlier by Finley (1966), Warren (1960) and Judson and Edgar (1963). These works were solely on the physical features of sonic counter flow injection to supersonic free stream. Important features of the flow field resulting due to counter flow injection in a sphere-cone-flare are shown in Fig. 1. The counter flow jet separates from the sharp edged orifice. The jet expands till a mach disc is formed and thereafter it turns in the direction of mainstream.

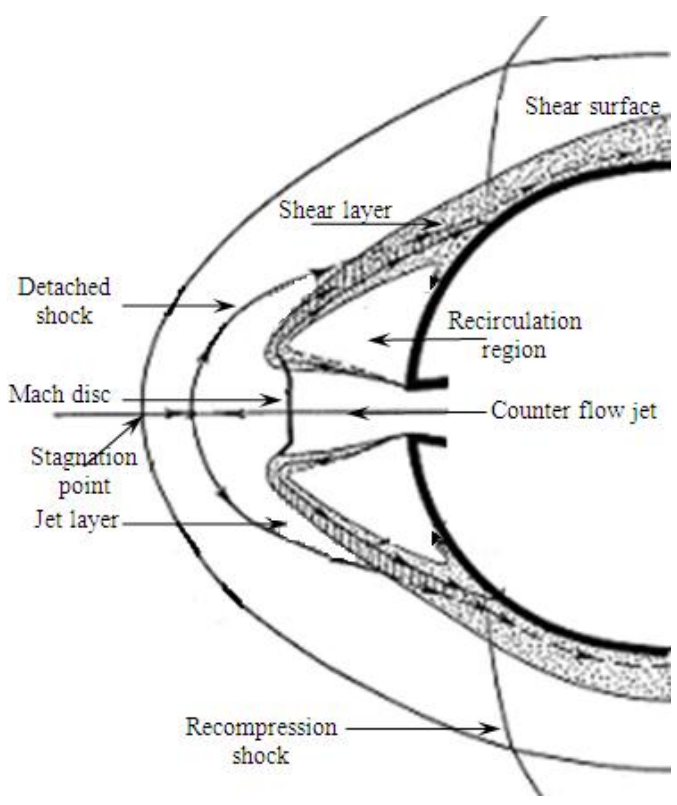

Fig. 1. Schematic of counter flow jet flow field

Due to the low pressure created by expansion of the jet, a recirculation region is formed in the shear layer. The jet layer mixes with shear layer of mainstream only after this recirculation region thereby forming a dividing stream surface. The jet layer turns along the body surface flows along downstream. The pressure rise associated with the reattachment of the shear layer causes a recompression shock in the jet layer and the flow from the mainstream outside the interface. Hence the shear surface turns downstream from the intersection of the recompression shock with the main bow shock.

\section{GOVERNING EQUATIONS}

The conservation form of equations, which govern 2D/axisymmetric turbulent compressible flow can be expressed in a generic form can be written as

$\frac{\partial U}{\partial t}+\frac{\partial F}{\partial x}+\frac{1}{r^{m}} \frac{\partial\left(r^{m} G\right)}{\partial y}=S$

Where $\mathrm{m}=0$ for $2-\mathrm{D}$ and $\mathrm{m}=1$ for axisymmetric problems

$U=\left[\begin{array}{l}\rho \\ \rho u \\ \rho v_{r} \\ \rho E \\ \rho \kappa \\ \rho \varepsilon\end{array}\right], \quad \mathrm{F}=\left[\begin{array}{l}\rho u \\ \rho u^{2}+\sigma_{x x} \\ \rho u v_{r}+\sigma_{x r} \\ \left(\rho E+\sigma_{x x}\right) u+\sigma_{x r} v_{r}-K_{\text {eff }} \frac{\partial T}{\partial x} \\ \rho u \kappa-\mu_{\kappa} \frac{\partial \kappa}{\partial x} \\ \rho u \varepsilon-\mu_{\varepsilon} \frac{\partial \varepsilon}{\partial x}\end{array}\right]$ 


$$
\left.\begin{array}{l}
\mathrm{G}=\left[\begin{array}{l}
\rho v_{r} \\
\rho u v_{r}+\sigma_{r x} \\
\rho v_{r}{ }^{2}+\sigma_{x r} \\
\left(\rho E+\sigma_{r r}\right) v_{r}+\sigma_{r x} u-K_{e f f} \frac{\partial T}{\partial r} \\
\rho v_{r} \kappa-\mu_{\kappa} \frac{\partial \kappa}{\partial r} \\
\rho v_{r} \varepsilon-\mu_{\varepsilon} \frac{\partial \varepsilon}{\partial r}
\end{array}\right] \text { and } S=\left[\begin{array}{l}
0 \\
0 \\
\frac{m}{r}\left(P+\sigma_{\theta \theta}\right) \\
0 \\
H_{\kappa} \\
H_{\varepsilon}
\end{array}\right] \\
\sigma_{x x}=P+\frac{2}{3} \rho \kappa-\mu_{e f f}\left[2 \frac{\partial u}{\partial x}-\frac{2}{3}\left(\frac{\partial u}{\partial x}+\frac{1}{r} \frac{\partial r v_{r}}{\partial r}\right)\right] \\
\sigma_{x r}=\sigma_{r x}=-\mu_{e f f}\left[\frac{\partial u}{\partial r}+\frac{\partial v_{r}}{\partial x}\right] \\
\sigma_{r r}=P+\frac{2}{3} \rho \kappa-\mu_{e f f}\left[2 \frac{\partial v_{r}}{\partial r}-\frac{2}{3}\left(\frac{\partial u}{\partial x}+\frac{1}{r} \frac{\partial r v_{r}}{\partial r}\right)\right] \\
\sigma_{\theta \theta}=-\mu_{e f f}\left[2 \frac{v_{r}}{r}-\frac{2}{3}\left(\frac{\partial u}{\partial x}+\frac{1}{r} \frac{\partial r v_{r}}{\partial r}\right)\right]
\end{array}\right]
$$

In the above versions of formulations, the effective viscosity of the flow field

$$
\mu_{\text {eff }}=\mu_{l}+\mu_{t}
$$

From Sutherland's law laminar viscosity is found as

$$
\mu_{l}=1.458 \times 10^{-6} \frac{T^{1.5}}{T+110.4}
$$

and $\mu_{t}$ is found from turbulence model. The effective thermal conductivity is

$$
K_{\text {eff }}=K_{l}+K_{t}
$$

In which the laminar and turbulent conductivities are

$k_{l}=\frac{\mu_{l} C p}{\operatorname{Pr}}$ and $k_{t}=\frac{\mu_{t} C p}{\operatorname{Pr}_{t}}$

For the present analysis modified $\kappa-\varepsilon$ model called Renormalisation Group (RNG) model was used. Yakhot et al. (1992) had proposed this model, which systematically removes all the small scales of turbulence motion from the governing equation by expressing their effects in terms of large scales and a modified viscosity. Following are the two equations related to RNG model.

$$
\begin{aligned}
& \frac{\partial(\rho \kappa)}{\partial t}+\operatorname{div}(\rho \kappa U)=\operatorname{div}\left[\alpha_{\kappa} \mu_{\text {eff }} \operatorname{grad} \kappa\right]+H_{\kappa} \\
& \frac{\partial(\rho \varepsilon)}{\partial t}+\operatorname{div}(\rho \varepsilon U)=\operatorname{div}\left[\alpha_{\kappa} \mu_{\text {eff }} \operatorname{grad} \varepsilon\right]+H_{\varepsilon}
\end{aligned}
$$

Here the turbulence source terms are obtained as given below

$$
H_{\kappa}=2 \mu_{t} E_{i j}-\rho \varepsilon \quad \text { and } \mathrm{H}_{\varepsilon}=C_{1 \varepsilon} * \frac{\varepsilon}{\kappa} 2 \mu_{t} E_{i j} \cdot E_{i j}-C_{2 \varepsilon} \rho \frac{\varepsilon^{2}}{\kappa}
$$

Turbulent viscosity is defined as $\mu_{t}=\rho C_{\mu} \frac{\kappa^{2}}{\varepsilon}$

Closure coefficients are evaluated as

$\mathrm{C}_{\mu}=0.0845, \alpha_{\mathrm{\kappa}}=\alpha_{\varepsilon}=1.39, \mathrm{C}_{1 \varepsilon}=1.42, \mathrm{C}_{2 \varepsilon}=1.68$

$\eta=\sqrt{\left(2 \mathrm{E}_{\mathrm{ij}} \cdot \mathrm{E}_{\mathrm{ij}}\right)^{\kappa / \varepsilon}}$ and $\mathrm{C}_{1 \varepsilon}{ }^{*}=C_{1 \varepsilon}-\frac{\eta\left(1-\eta / \eta_{0}\right)}{1+\beta \eta^{3}}$

$\eta_{0=} 4.377, \beta=0.012$.

Value of constant $\beta$ is found from near wall turbulence data. All other parameters are explicitly computed as part of the RNG calculations.

\section{NUMERICAL METHOD}

The finite volume method is compatible enough to handle unstructured grids, if information regarding the neighboring elements is supplied. In this analysis, the domain is divided in to quadrilateral control volumes.

The basic conservation equation is

$$
\frac{\partial U}{\partial t}+\nabla \cdot F-S=0
$$

Integrating this equation,

$\int\left(\frac{\partial U}{\partial t}+\nabla \cdot F-S\right) d \Omega=0$

i.e. $\int \frac{\partial U}{\partial t} d \Omega+\int \nabla \cdot F d \Omega+\int S d \Omega=0$

Applying greens theorem

$\int_{\Omega} \frac{\partial U}{\partial t} d \Omega+\int_{\Gamma} F n d \Gamma-\int_{\Omega} S . d \Omega=0$

Where $\Gamma$ and $\Omega$ are respectively the surface area and volume of the cell. The above equation can be rewritten as

$$
\frac{d U_{i}}{d t} V_{i}+\sum_{\text {faces }} F \cdot d s-S_{i .} V_{i}=0
$$

Here $\mathrm{Vi}$ is the cell volume and $\mathrm{ds}$ is the area of elemental sides. In all AUSM schemes inviscid flux is split into convective and pressure fluxes

$$
F=F c+P=\dot{m} \vec{\psi}+P
$$

The convective terms can be considered as passive scalar quantities convected by a suitably defined velocity at the cell interface. Pressure flux terms are governed by the acoustic wave speeds. The numerical flux at a cell interface for AUSM+-UP with appropriate numerical speed of sound is given in the work of Liou (1996).

$$
F_{\frac{1}{2}, A U S M+}=\left(\left.M_{L}^{+}\right|_{\beta=\frac{1}{8}}+\left.M_{R}^{-}\right|_{\beta=1 / 8}\right)_{c_{1} / 2} \psi_{L}+\left(\left.P_{L}^{+}\right|_{\alpha=3 / 16}+\left.P_{R}^{-}\right|_{\alpha=3 / 16}\right) P_{R}
$$$$
\text { If } M_{\frac{1}{2}}=M_{L}^{+}+M_{R}^{-}>0
$$

For the simple explicit scheme the time stepping using Runge-Kutta method are as given below 


$$
\begin{aligned}
& U_{i}^{(0)}=U_{i}^{(n)} \\
& U_{i}^{(1)}=U_{i}^{(0)}-\alpha_{1} \frac{\Delta t_{i}}{V_{i}}\left(R_{i}^{(0)}\right) \\
& U_{i}^{(2)}=U_{i}^{(0)}-\alpha_{2} \frac{\Delta t_{i}}{V_{i}}\left(R_{i}^{(1)}\right) \\
& U_{i}^{(3)}=U_{i}^{(0)}-\alpha_{3} \frac{\Delta t_{i}}{V_{i}}\left(R_{i}^{(2)}\right) \\
& U_{i}^{n+1}=U_{i}^{n}+U_{i}^{(3)}
\end{aligned}
$$

Where the superscripts $n$ and $n+1$ indicate the current and the next time steps. The values of coefficients in Runge-Kutta integration procedure are $\alpha_{1=0} 0.6, \alpha_{2=} 0.6$ and $\alpha_{3}=1.0$. This method is only conditionally stable, as it is an explicit method. Local time stepping is employed for accelerating convergence. Thus each control volume can march with its own maximum allowable time step specified by the explicit stability criteria given by

$$
\Delta t_{i} \leq \frac{\Delta l_{i}}{q_{i}+c}
$$

where $q_{i}$ is the magnitude of fluid velocity of $\mathrm{i}^{\text {th }}$ cell, given by $q_{i}=\sqrt{u_{i}^{2}+v_{i}^{2}}$ and $c=\sqrt{\gamma R T}$, the sound velocity and $\Delta l_{i}$ is the characteristic dimension of the quadrilateral element. Now the time step of the explicit solution procedure is given by

$$
\Delta t_{i}=C F L \times \frac{\Delta l_{i}}{q_{i}+c}
$$

where CFL is the Courant, Friedrichs and Lewis number.

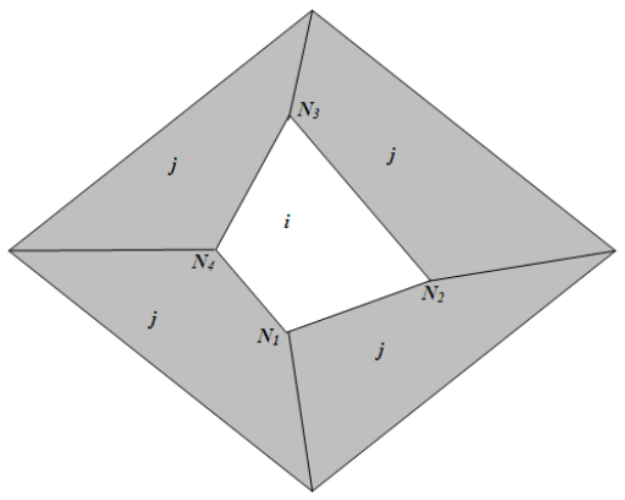

Fig. 2. Control volume and its neighbours

Solution mapped least square based higher order reconstruction scheme is used in this solver. Consider a computational domain discretized into quadrilateral control volumes each having a volume $V_{i}$ and a

geometric centroid $\left(x_{i}, y_{i}\right)$. Let $\phi(x, y)$ be any function defined over the control volume with an average value of $\bar{\phi}_{i}$ for $i^{\text {th }}$ control volume. Let $\Delta x_{i}=x-x_{i}$ and $\Delta y_{i}=y-y_{i}$. The aim is to find a polynomial $\mathrm{P}\left(\Delta x_{i}, \Delta y_{i}\right)$ that can be used for higher order reconstruction. The reconstructing polynomial $\mathrm{P}\left(\Delta x_{i}, \Delta y_{i}\right)$ can be expressed as

$$
\begin{aligned}
& \mathrm{P}\left(\Delta x_{i}, \Delta y_{i}\right)=\left.\phi\right|_{x_{i}, y_{i}}+\left.\frac{\partial \phi}{\partial x}\right|_{x_{i}, y_{i}} \Delta x_{i}+\left.\frac{\partial \phi}{\partial y}\right|_{x_{i}, y_{i}} \Delta y_{i}+\left.\frac{\partial^{2} \phi}{\partial x^{2}}\right|_{x_{i}, y_{i}} \frac{\Delta x_{i}^{2}}{2} \\
& +\left.\frac{\partial^{2} \phi}{\partial x \partial y}\right|_{x_{i}, y_{i}} \Delta x_{i} \Delta y_{i}+\left.\frac{\partial^{2} \phi}{\partial y^{2}}\right|_{x_{i}, y_{i}} \frac{\Delta y_{i}^{2}}{2}+\left.\frac{\partial^{3} \phi}{\partial x^{3}}\right|_{x_{i}, y_{i}} \frac{\Delta x_{i}^{3}}{6} \\
& +\left.\frac{\partial^{3} \phi}{\partial x^{2} \partial y}\right|_{x_{i}, y_{i}} \frac{\Delta x_{i}^{2} \Delta y_{i}}{2}+\left.\frac{\partial^{3} \phi}{\partial x \partial y^{2}}\right|_{x_{i}, y_{i}} \frac{\Delta x_{i} \Delta y_{i}^{2}}{2}+\left.\left.\frac{\partial^{3} \phi}{\partial y^{3}}\right|_{x_{i}, y_{i}} \frac{\Delta y_{i}^{3}}{\partial x^{4}}\right|_{x_{i}, y_{i}} \frac{\Delta x_{i}^{4}}{24}+\left.\frac{\partial^{4} \phi}{\partial x^{3} \partial y}\right|_{x_{i}, y_{i}} \frac{\Delta x_{i}^{3} \Delta y_{i}}{6}+\left.\frac{\partial^{4} \phi}{\partial x^{2} \partial y^{2}}\right|_{x_{i}, y_{i}} \frac{\Delta x_{i}^{2} \Delta y_{i}^{2}}{4} \\
& +\left.\frac{\partial^{4} \phi}{\partial x \partial y^{3}}\right|_{x_{i}, y_{i}} \frac{\Delta x x_{i} \Delta y_{i}^{3}}{6}+\left.\frac{\partial^{4} \phi}{\partial y^{4}}\right|_{x_{i}, y_{i}} \frac{\Delta y_{i}^{4}}{24}
\end{aligned}
$$

The mean of any property has to be conserved for any control volume. Therefore

$$
\int_{V_{i}} \phi(x, y) d V=\int_{V_{i}} \mathrm{P}\left(\Delta x_{i}, \Delta y_{i}\right) d V=\bar{\phi}_{i}
$$

Thus $\bar{\phi}_{i}$ is obtained by integrating $\mathrm{P}\left(\Delta x_{i}, \Delta y_{i}\right)$ over the control volume. That is

$$
\begin{aligned}
& \overline{\phi_{i}}=\left.\phi\right|_{x_{i}, y_{i}}+\left.\frac{\partial \phi}{\partial x}\right|_{x_{i}, y_{i}} \bar{x}+\left.\frac{\partial \phi}{\partial y}\right|_{x_{i}, y_{i}} \bar{y}+\left.\frac{\partial^{2} \phi}{\partial x^{2}}\right|_{x_{i}, y_{i}} \frac{\overline{x^{2}}}{2} \\
& +\left.\frac{\partial^{2} \phi}{\partial x \partial y}\right|_{x_{i}, y_{i}} \overline{x y}+\left.\frac{\partial^{2} \phi}{\partial y^{2}}\right|_{x_{i}, y_{i}} \frac{\overline{y^{2}}}{2}+\left.\frac{\partial^{3} \phi}{\partial x^{3}}\right|_{x_{i}, y_{i}} \frac{\overline{x^{3}}}{6}+ \\
& \left.\frac{\partial^{3} \phi}{\partial x^{2} \partial y}\right|_{x_{i}, y_{i}} \frac{\overline{x^{2} y}}{2}+\left.\frac{\partial^{3} \phi}{\partial x \partial y^{2}}\right|_{x_{i}, y_{i}} \frac{\overline{x y^{2}}}{2}+\left.\frac{\partial^{3} \phi}{\partial y^{3}}\right|_{x_{i}, y_{i}} \frac{\overline{y^{3}}}{6} \\
& +\left.\frac{\partial^{4} \phi}{\partial x^{4}}\right|_{x_{i}, y_{i}} \frac{\overline{x^{4}}}{24}+\left.\frac{\partial^{4} \phi}{\partial x^{3} \partial y}\right|_{x_{i}, y_{i}} \frac{\overline{x^{3} y}}{6}+\left.\frac{\partial^{4} \phi}{\partial x^{2} \partial y^{2}}\right|_{x_{i}, y_{i}} \frac{\overline{x^{2} y^{2}}}{4}+ \\
& \left.\frac{\partial^{4} \phi}{\partial x \partial y^{3}}\right|_{x_{i}, y_{i}} \frac{\overline{x y^{3}}}{6}+\left.\frac{\partial^{4} \phi}{\partial y^{4}}\right|_{x_{i}, y_{i}} \frac{\overline{y^{4}}}{24}
\end{aligned}
$$

Squaring the error between reconstructed value of $j^{\text {th }}$ neighbour of $i^{\text {th }}$ control volume and actual value of $\phi_{j}$

$$
\varepsilon_{i j}^{2}=\left(\frac{1}{V_{j}} \int_{V_{j}} \mathrm{P}\left(\Delta x_{i}, \Delta y_{i}\right) d V-\phi_{j}\right)^{2}
$$

Applying least square principle

$$
\begin{aligned}
& \frac{\partial \varepsilon_{i j}^{2}}{\partial \phi}=\frac{\partial \varepsilon_{i j}^{2}}{\partial(\partial \phi / \partial x)}=\frac{\partial \varepsilon_{i j}^{2}}{\partial(\partial \phi / \partial y)}=\frac{\partial \varepsilon_{i j}^{2}}{\partial\left(\partial^{2} \phi / \partial x^{2}\right)}=\frac{\partial \varepsilon_{i j}^{2}}{\partial\left(\partial^{2} \phi / \partial x \partial y\right)} \\
& =\frac{\partial \varepsilon_{i j}^{2}}{\partial\left(\partial^{2} \phi / \partial y^{2}\right)}=\frac{\partial \varepsilon_{i j}^{2}}{\partial\left(\partial^{3} \phi / \partial x^{3}\right)}=\frac{\partial \varepsilon_{i j}^{2}}{\partial\left(\partial^{3} \phi / \partial x^{2} \partial y\right)}=\frac{\partial \varepsilon_{i j}^{2}}{\partial\left(\partial^{3} \phi / \partial x y^{2}\right)} \\
& =\frac{\partial \varepsilon_{i j}^{2}}{\partial\left(\partial^{3} \phi / \partial y^{3}\right)}=\frac{\partial \varepsilon_{i j}^{2}}{\partial\left(\partial^{4} \phi / \partial x^{4}\right)}=\frac{\partial \varepsilon_{i j}^{2}}{\partial\left(\partial^{4} \phi / \partial x^{3} y\right)}=\frac{\partial \varepsilon_{i j}^{2}}{\partial\left(\partial^{4} \phi / \partial x^{2} y^{2}\right)} \\
& =\frac{\partial \varepsilon_{i j}^{2}}{\partial\left(\partial^{4} \phi / \partial x y^{3}\right)}=\frac{\partial \varepsilon_{i j}^{2}}{\partial\left(\partial^{4} \phi / \partial y^{4}\right)}=0
\end{aligned}
$$


After applying the least square principle, the resulting set of equations can also be written in the matrix form as

$$
\left[A_{i j}\right][\Delta \phi]=\left[A_{i} \phi_{j}\right]
$$

Where the matrices are given by

$$
\begin{aligned}
& {\left[A_{i j}\right]=\left[\begin{array}{cccc}
1 & 0 & 0 & - \\
\sum_{j=1}^{N} A_{1} & \sum_{j=1}^{N}\left(A_{1} A_{1}\right) & \sum_{j=1}^{N}\left(A_{1} A_{2}\right) & - \\
\sum_{j=1}^{N} A_{2} & \sum_{j=1}^{N}\left(A_{2} A_{1}\right) & \sum_{j=1}^{N}\left(A_{2} A_{2}\right) & - \\
- & - & - & -
\end{array}\right]} \\
& {[\Delta \phi]=\left[\begin{array}{llll}
\phi_{i} & \frac{\partial \phi_{i}}{\partial x} & \frac{\partial \phi_{i}}{\partial y} & -
\end{array}\right]^{T}} \\
& {\left[A_{i} \phi_{j}\right]=\left[\begin{array}{llll}
\phi_{j} & \sum_{j=1}^{N} A_{1} \phi_{j} & \sum_{j=1}^{N} A_{2} \phi_{j} & \sum_{j=1}^{N} B_{1} \phi_{j}-
\end{array}\right]^{T}}
\end{aligned}
$$

Geometric weights [] or the solution independent weights are evaluated based on the distance between the control volume $i$ under consideration and the neighbouring control volume $j$ is given by

$$
\omega_{i j}=\frac{1}{\Delta x_{j i}^{2}+\Delta y_{j i}^{2}}
$$

\section{SOLVER VALIDATION}

\subsection{Hypersonic flow over blunted cone-flare}

Flow field around blunted cone-flare is of particular interest since it features most of the aspects of the hypersonic flow around re-entry vehicles. The region between the cone and the flare is critical with respect to the evaluation of the surface heat flux. Flow separation is induced by the shock wave-boundary layer interactions, with subsequent flow reattachment that can dramatically enhance the surface heat transfer.

The experiment was carried out in $\mathrm{H} 3$ hypersonic wind tunnel (Savino and Paterna, 2005) and the chamber conditions ensure that the flow is purely laminar. Hence this is one of the most suited test cases for validating various schemes of the solver since uncertainties due to turbulence are eliminated.

The geometry and computational domain of the blunted cone-flare is shown in Fig. 3. Conditions at inlet (extreme left) are imposed in the form of a supersonic jet. Static pressure $P$, static temperature $T$ and free stream Mach number $M \propto$ were specified at this boundary.

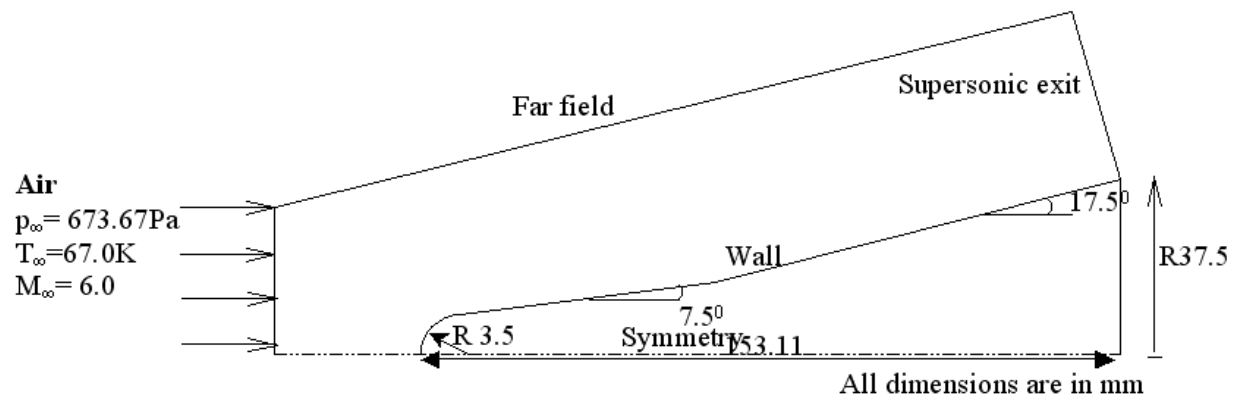

Fig. 3. Computational domain and boundary conditions

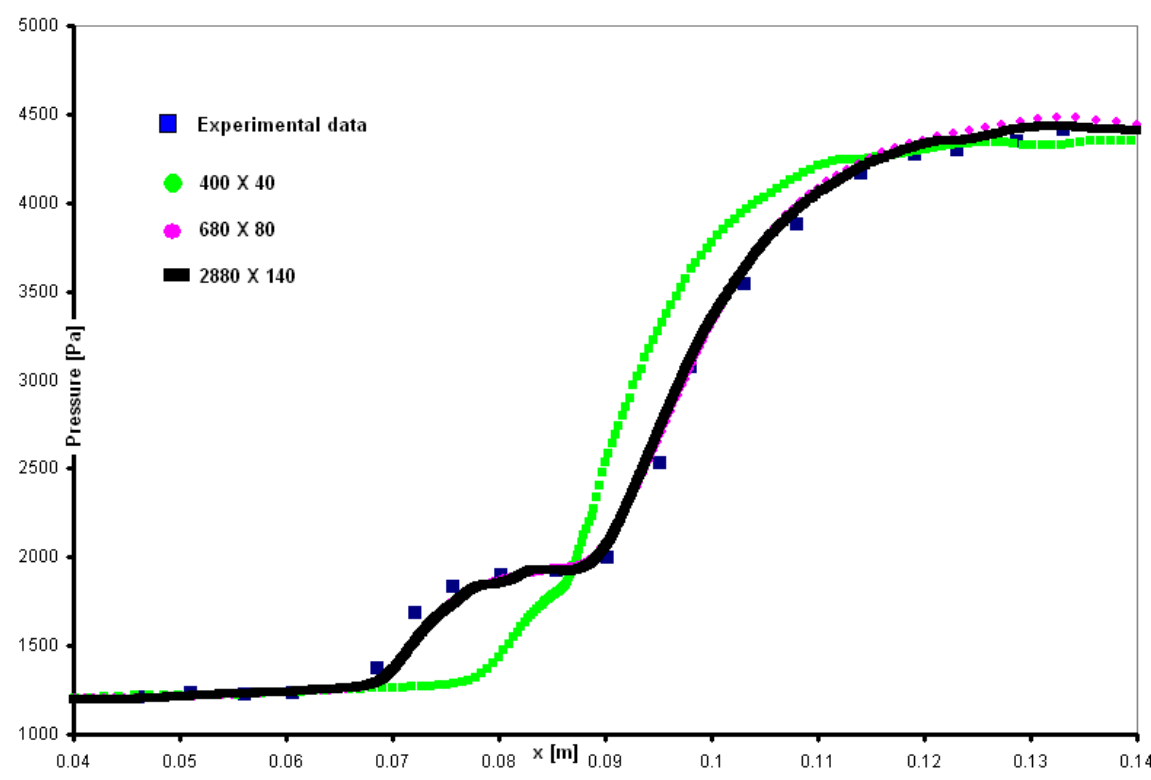

Fig. 4. Wall pressure distribution 
Other variables are evaluated from local isentropic relations. Adiabatic no slip conditions (i.e. $\mathrm{u}=\mathrm{v}=\mathrm{w}=$ $0, \frac{\partial T}{\partial n}=0$ ) were used at solid wall, as this is the numerical boundary of viscous flows. For supersonic outflow, the properties (static pressure, static temperature and velocities) are linearly interpolated at the exit nodes.

Air at $580 \mathrm{~K}$ and 32 bar is passed through an axisymmetric nozzle where it expands to a nominal Mach number of 6 and enters the test section. The stagnation pressure is $10 \mathrm{bar}$ and the unit length Reynolds number is $8 \times 10^{6} \mathrm{~m}^{-1}$. Under these conditions flow over the entire blunted cone-flare is laminar.

Simulations were carried out using constant wall temperature of $300 \mathrm{~K}$. Grid independent solutions were obtained for $2800 \times 140$ grids. The comparison of computed wall pressure distribution with experiment for different grids is shown in Fig. 4. Coarse mesh is not capable of capturing the separation region in the cone-flare junction exactly. Refined mesh successfully predicts the wall pressure in all regions. Comparison of numerical and empirical shock shapes (Billig, 1967) is given in Fig. 5. It can be seen that the bow shock is getting weakened as it departs from stagnation point and both the shock shapes shows reasonably good match. Mach number contour plot corresponding to finest grid is shown in Fig. 6. All features such as detached bow shock in stagnation region and shockboundary layer interaction in cone-flare junction are captured.

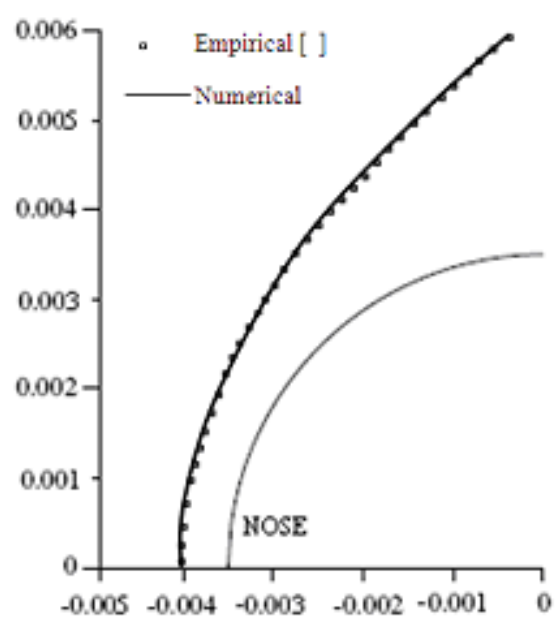

Fig. 5. Comparison of numerical and empirical shock shape

\subsection{Opposing jet in hypersonic flow}

Experimental results of Aso et al. (2002) on opposing jet in hypersonic flow are used to validate the present code. Sonic nitrogen injection is introduced through a hole of diameter $4 \mathrm{~mm}$ in the opposite direction of supersonic frees stream $(M=3.96)$. The diameter of the blunt model is $50 \mathrm{~mm}$ and the Reynolds number based on this diameter of blunt body is $2.1 \times 10^{6}$. Total pressure ratio of jet to free stream is maintained as 0.276 . The computational domain and test conditions are summarized in Fig. 7.

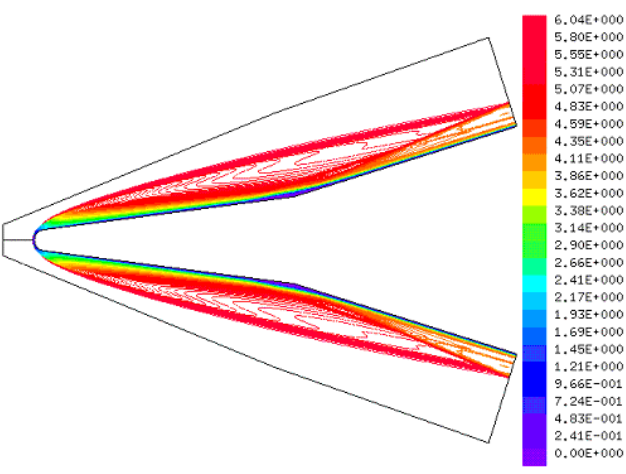

Fig. 6. Mach contours

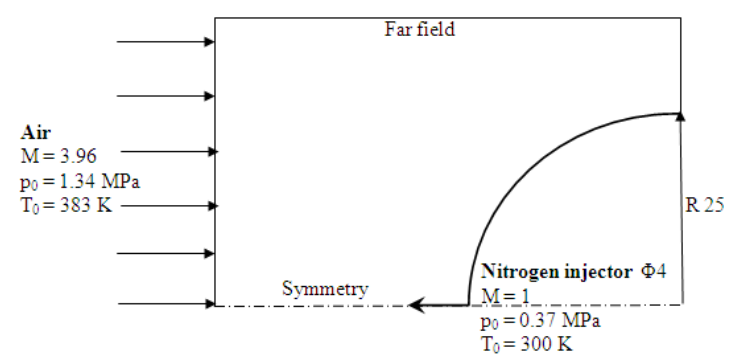

Fig. 7. Computational domain and test conditions

The computational domain is discretized into 120000 cells with two sets of grid blocks. A fine block of grid introduced near wall extends $20 \mathrm{~mm}$ above wall. Minimum grid size near wall is $0.01 \mathrm{~mm}$. Boundary conditions used are the same pressure, temperature and Mach number as given earlier in the experimental data. A supersonic inflow condition was maintained at the left face and outflow condition is maintained at right face of the computational domain. No-slip wall condition is given for blunt body wall except at portion of jet, where jet is introduced as a constant velocity condition.

Entire flow field is initialised with the free stream conditions and the solution is marched in the time with an initial CFL of 0.01 , which was gradually increased to 0.6. The criterion for convergence of the solution is selected as the density error in successive iterations, which has to become less than 1.0E-06. After about 22,000 iterations at CFL value equal to 0.6, the convergence has been observed. Mach number contour plot of the computed results is shown in Fig. 8. All important features of the flow field resulting due to counter flow injection in a sphere-cone-flare, illustrated in section 2, have been successfully captured. The formation of Mach disc, recirculation region formed due to expansion of jet, detached shock wave, recompression shock etc can be visualized in this plot. Computation of another test on same blunt body without injection is done in order to show the effect of gas jet on aerodynamic heating.

Mach number contour plot of this computed result is shown in Fig. 9. Experimental data for wall pressure are available for both cases; computed results are compared with it and are shown in Fig. 10. It is evident from the plot that the numerical scheme used is capable of 
predicting the flow behavior. Computed pressure value near to recirculation region (injection case) deviates slightly due to the presence of associated unsteadiness. Also numerical result of Aso et al (2002) has also a similar trend for the pressure variation.

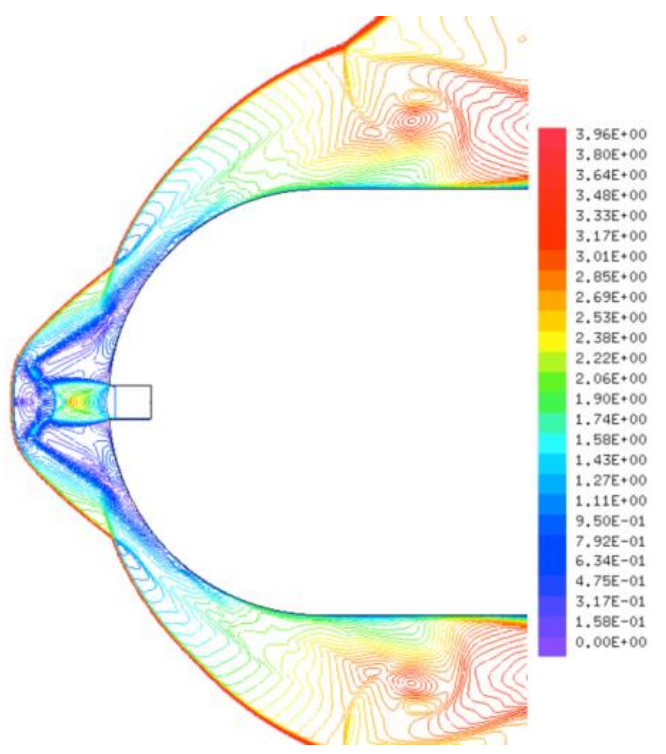

Fig. 8. Mach number contours for test case with injection

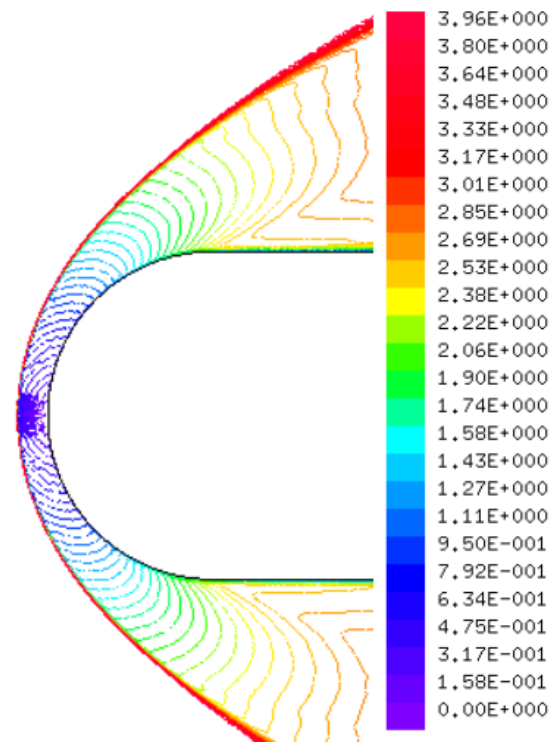

Fig. 9. Mach number contours for test case without injection

Pressure reduction on nose of blunt body resulting due to introduction of jet can also be observed. Figure 11 compares the computed wall temperature distribution with and without injection. It can be observed that the injected stream confines in wall region of the blunt body and reduces the aerodynamic heating due to free stream. More cooling effect is observed near nose of the blunt body due to the presence of strong recirculation, wherein maximum temperature is expected without injection.

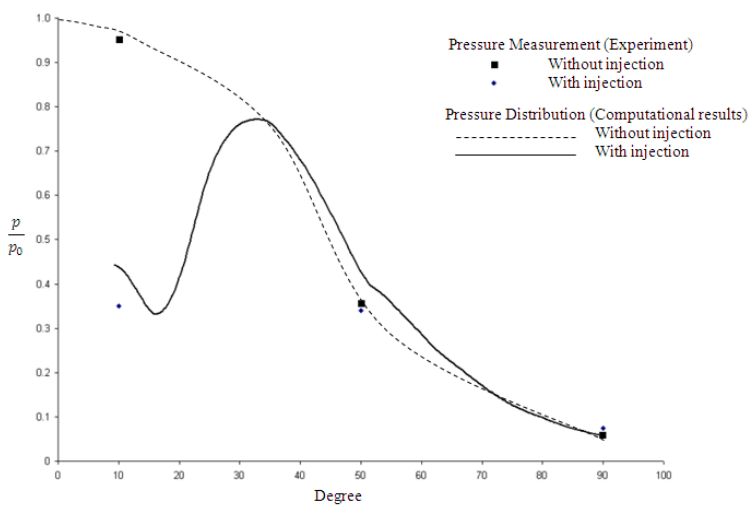

Fig. 10. Comparison of computed wall pressure distribution

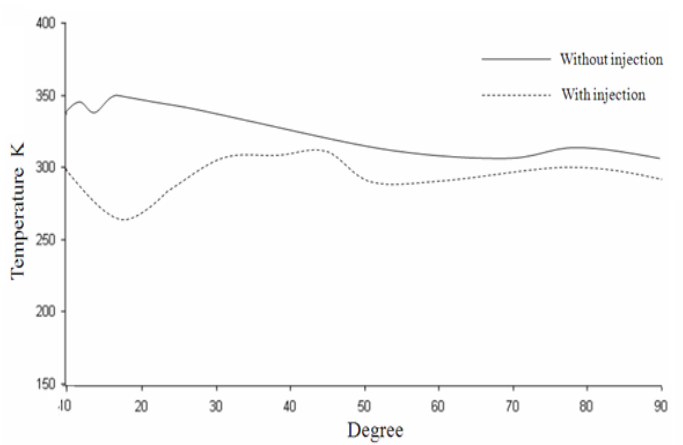

Fig. 11. Comparison of computed wall temperature distribution with and without injection

\section{EFFECT OF FILM COOLING FOR A HYPERSONIC REENTRY MODULE}

The present solver is used to study the effect of film cooling due to a counter flow jet on a hypersonic reentry module flying at Mach 8 . The free stream total pressure is $2217 \mathrm{kPa}$, total temperature is $3600 \mathrm{~K}$ and that of the jet are 0.4 bar and $300 \mathrm{~K}$ respectively. The computational domain is discretized into $1,32,000$ control volumes with two sets of grid blocks. A fine block of grid introduced near wall extends $20 \mathrm{~mm}$ above wall. Minimum grid size near wall is $0.01 \mathrm{~mm}$. Entire flow field is initialized with above said conditions and marched in time with an initial CFL of 0.01 , which was gradually increased to 0.5 . The criterion for convergence of the solution is selected as the density error in successive iterations, which has to become less than 1.0E-06. The convergence has been observed after about 30,000 iterations at CFL value equal to 0.5.Mach number field plot is shown in Fig. 12. The presence of a strong bow shock in front of the nose can be observed. Free stream Mach number 8 is reduced to 4 across the bow shock wave. Other important flow features such as Mach disc, recompression shock wave, and recirculation in jet expansion region can also be observed in this plot.

The contour plot of temperature is given in Fig. 13. It is observed that the high temperature region is kept away from wall and this significantly reduces wall heat flux. 
The position of the bow shock is indicated by the high temperature contour is situated at larger standoff distance from the nose cap. The cold counter jet flow emanating from the nose tip gets decelerated downstream the Mach disk as it approaches the free stream and turns near to wall. Thus a separation boundary layer of cold air is formed, which protects the surface of the module from aerodynamic heating.

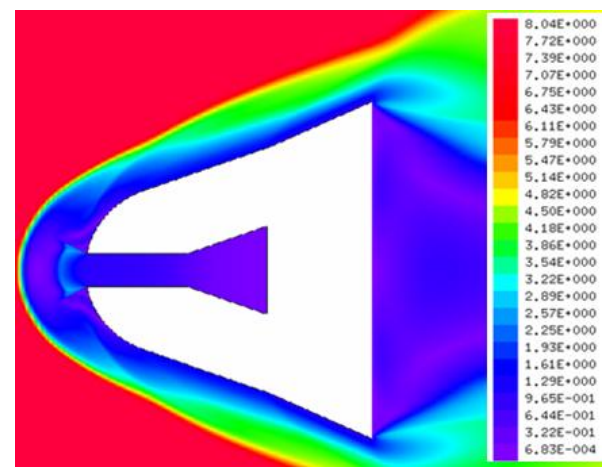

Fig. 12. Field view of Mach number around hypersonic reentry module

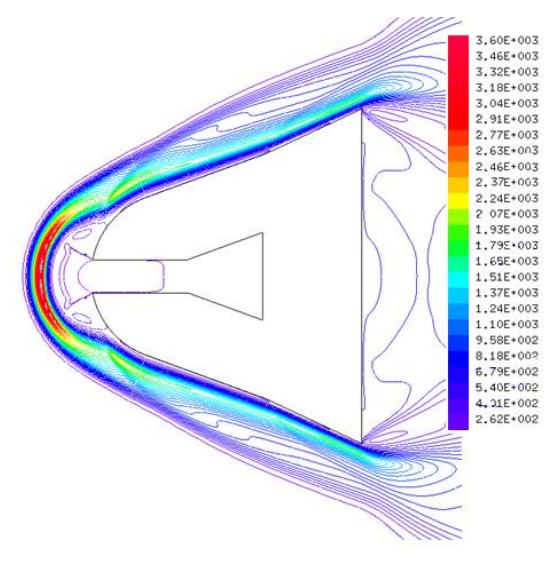

Fig. 13. Temperature contour plot for film cooling on hypersonic reentry module

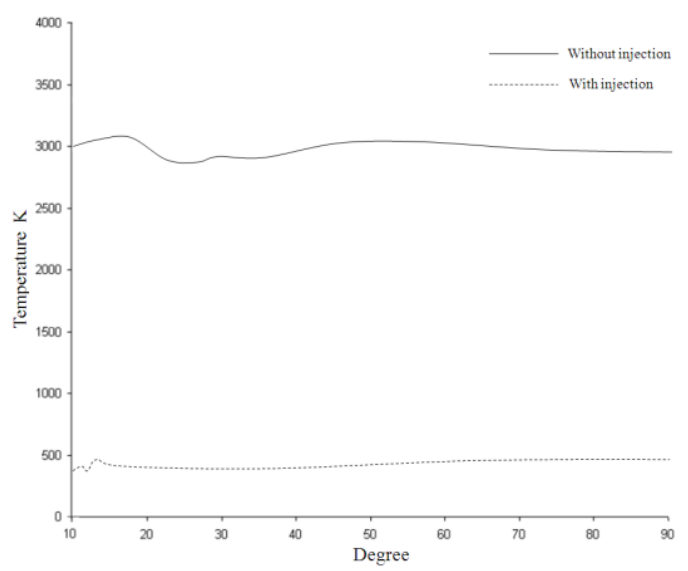

Fig. 14. Comparison of computed wall temperature distribution with and without injection
A comparison of computed wall temperature distribution is given in Fig. 14. The effect of making a cold layer on wall by the injected stream is evident from this comparison. Reentry modules may have free space where gas to be injected can be stored. A full scale model can be tested in a similar fashion in order to identify the multiple locations and quantity of injectors needed for complete protection.

\section{Conclusions}

A numerical code based on AUSM+-UP scheme using higher order accurate reconstruction method has been developed and successfully validated. Evaluation of convective fluxes using AUSM+-UP scheme and derivative evaluation using least square based reconstruction scheme was quite successful in simulating the complex features associated with film cooling of a hypersonic re-entry module.

The AUSM +-UP performs well and all discontinuities in flow field are successfully captured. The present study establishes the effect of film cooling due to opposed jet on a blunt body in hypersonic flow. A significant reduction in wall temperature due to aerodynamic heating is observed. The developed flow solver could reveal all features of the flow field and computed results had good agreement with experimental results. These numerical results provides vital information required for the design of effective cooling systems such as number of injectors, their dimensions and locations, injection pressure and temperature, mass flow rate required etc. Hence it helps significantly enhancing the aerodynamic characteristics and aero thermal performance of supersonic and hypersonic vehicles and reentry modules

\section{REFERENCES}

Aso, S., K. Hayashi and M. Mizoguchi (2002). A Study on Aerodynamic Heating Reduction due to Opposing Jet in Hypersonic Flow. AIAA 20020646.

Billig, F.S. (1967). Shock-wave shapes around spherical- and cylindrical-nosed bodies. Journal of Spacecraft Rockets 4, 822-823.

David, J.R. and R. James (1963). Exploratory investigation of the effect of a forward facing jet on the bow shock of a blunt body in a Mach number of 6 free stream. NASA TN D-1605.

Finley, P.J. (1966). The flow of a jet from a body opposing a supersonic free stream. Journal of Fluid Mechanics 26, 337-368.

Liou, M.S. (2006). A sequel to AUSM, Part II: AUSM+-UP for all speeds. Journal of Computational Physics 214, 137-170.

Liou, M.S. (1996). A sequel to AUSM: AUSM+. Journal of Computational Physics 129, 364-382. 
P. Nair et al. / JAFM, Vol. 3, No. 1, pp. 15-23, 2010.

Judson, R.B. and A. Edgar (1963). Experimental investigation of a two layer shock cap due to blunt body nose injection. Journal of Fluid Mechanics $15,442-448$.

Mc-Bride, B.J. and S. Gordon (1971). Computer Program for Calculation of Complex Chemical Equilibrium Compositions, Rocket Performance, Incident and Reflected Shocks, and ChapmanJouget Detonations. NASA SP-27371.

Sakagoshi, A. and M. Ken (2000). Effectiveness of an aerospike for reduction of hypersonic aerodynamic heating. ISTS 2000-e-23.

Savino R. and D. Paterna (2005). Blunted cone-flare in hypersonic flow. Computers and Fluids 34, 859875.

Warren, C.H.E. (1960). An experimental investigation of the effect of ejecting a coolant gas at the nose of a bluff body. Journal of Fluid Mechanics 8, 400417.

Yakhot, V., A. Orzag, T.B. Gatski and C.B. Speziade (1992). Development of Turbulence Model for Shear Flows by a Double Expansion Technique, Physics of Fluids 4, 1510-1520. 